Danielle Desmarais André Royal

\section{Filaments intermédiaires et expression génétique neuronale}

Les gènes qui encodent les différentes protéines des filaments intermédiaires sont exprimés selon un patron précis au cours du développement du système nerveux. Ainsi, le gène de la périphérine n'est exprimé que dans les neurones, et tout particulièrement dans ceux du système nerveux périphérique. L'expression de ce gène a été associée à la différenciation terminale des neurones. Le promoteur du gène de la périphérine présente une activité histospécifique et cette spécificité neuronale semble reposer sur un élément de régulation appelé PER1 qui chevauche la boîte TATA. Une protéine uniquement nucléaire présente dans les cellules qui expriment la périphérine se lie à l'élément PER1 et elle semble représenter un nouveau facteur de transcription spécifique à une sous-population de neurones. Différents mécanismes pourraient expliquer le rôle de PER1 dans la restriction histospécifique de l'expression génétique.

\section{ADRESSE}

D. Desmarais : Boursière du Conseil de recherches médicales du Canada.

A. Royal : Coordonnateur du Groupe de recherche en oncogénétique, titulaire de la chaire Hans Selye et chercheur boursier du Fonds de la recherche en santé du Québec. Département de pathologie, Université de Montréal. C.P. 6128 , succursale A. Montréal, Québec, Canada, $\mathrm{H} 3 \mathrm{C} 3 \mathrm{~J} 7$
La périphérine, une protéine de la famille des filaments intermédiaires, et le contrôle de l'expression génétique neuronale

Le développement embryonnaire et la différenciation cellulaire font partie des processus hyper-complexes. Par exemple, l'élaboration du système nerveux repose notamment sur la différenciation d'une grande variété de neurones qui doivent en se dévelop- pant trouver très précisément leur place et établir des connexions adéquates. On dispose de peu de connaissances sur la formation des neurones spécialisés. On sait cependant que l'expression d'un ensemble de gènes spécifiques, fruits d'une combinatoire particulière de facteurs de transcription, entre certainement en jeu. Les facteurs de transcription jouent un très grand rôle dans l'orchestration des processus du développement. Ainsi, l'expression des gènes propres aux cellules musculaires, érythroïdes ou hépatiques sont placés sous le contrôle de facteurs de 
transcription histospécifiques [1, 2, 3]. Pour saisir la subtilité des mécanismes moléculaires qui concourent au développement et à la différenciation neuronale, de nombreuses équipes de recherche privilégient l'analyse des promoteurs des gènes qui caractérisent les divers types de neurones. Parmi les gènes exprimés dans le système nerveux, ce sont les gènes des protéines des filaments intermédiaires qui retiennent ici notre attention.

\section{Les filaments intermédiaires et la différenciation}

La plupart des cellules eucaryotes sont dotées d'un réseau de filaments intermédiaires (FI) dont la taille et la diversité les distinguent des autres filaments cytoplasmiques (microfilaments et microtubules). Les protéines des FI font partie d'une famille de plus de 40 protéines. Les polypeptides qui composent les FI, ont un domaine central $\alpha$-hélicoïdal qui comprend une sous-structure en heptade de la forme $(a-b-c-d-e-f-g)_{n}$ où des acides aminés apolaires occupent les positions a et $d$. La taille, la structure secondaire et la séquence du domaine central sont très conservées. Par contre, on observe des variations importantes dans les régions aminoterminale (tête) et carboxy-terminale (queue) (figure 1). Sur la base des homologies de leur séquence et de leur structure, les protéines des FI ont été subdivisées en 7 types dont l'expression correspond à des types de différenciation cellulaire spécifiques (Tableau I). La signification de cette diversité en termes de fonctions n'est pas connue. Par contre, l'expression des FI est une propriété fondamentale des cellules différenciées. Ainsi, les kératines sont exprimées dans les cellules épithéliales, la vimentine dans les cellules mésenchymateuses, la desmine dans les cellules des muscles, et ainsi de suite [4].

Dans les neurones, toutefois, la situation est plus complexe. Outre le triplet de neurofilaments présent dans la plupart des neurones [4], on retrouve dans différentes sous-populations neuronales plusieurs autres protéines de la famille des FI. Ces protéines comprennent la nestine dans les cellules

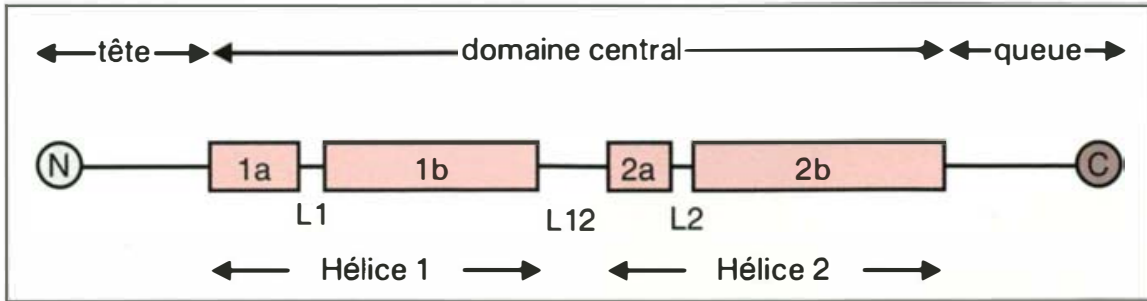

Figure 1. Organisation structurale des protéines de la famille des filaments intermédiaires. Le domaine central très conservé est flanqué par les régions hypervariables de tête et de queue situés aux extrémités amino (N) et carboxy (C)-terminales respectivement. Les éléments $\alpha$-hélicoïdaux 11a, 1b, $2 a$ et $2 b)$ sont séparés par les segments $L 1, L 2$ et $L 12$.

souches du neuroépithélium [5], l' $\alpha$ internexine dans certains neurones du système nerveux central (SNC) [6], la tanabine, exprimée entre autres dans le cône de croissance [7], la plasticine dans les cellules ganglionnaires de la rétine chez le poisson rouge [8] ; enfin, la périphérine dans le système nerveux périphérique (SNP) et dans quelques neurones du SNC [9] (Tableau I). La découverte de ces protéines particulières aux neurones soulève plusieurs questions, notamment sur leur fonction respective et sur le contrôle de leur expression. La diversité des FI neuronaux donne à penser qu'ils jouent un rôle dans l'organisation de la cellule différenciée. Au cours de la différenciation neuronale, la distribution intracellulaire et la composition du réseau de FI subissent des modifications qui les conduisent à adopter un arrangement définitif dans la cellule parvenue à maturité. Dans les cellules du neuroépithélium la nestine est remplacée par les FI typiques des cellules neuronales différenciées qui en dériveront (astrocytes, oligodendrocytes et neurones). L' $\alpha$ internexine, exprimée fortement dans le sytème nerveux embryonnaire, est réprimée dans le SNP adulte. Enfin, la périphérine ne sera induite que lorsque les neurones auront atteint leur position définitive. Parmi toutes ces protéines, la périphérine constitue un modèle qui présente un intérêt appréciable dans l'étude du contrôle de l'expression des gènes histospécifiques à cause des particularités de son expression dans l'animal et de la disponibilité de systèmes expérimentaux bien caractérisés permettant des études détaillées in vitro.

\begin{tabular}{|c|c|c|}
\hline \multicolumn{3}{|c|}{$\begin{array}{c}\text { DISTRIBUTION DES DIFFÉRENTS TYPES DE PROTÉINES } \\
\text { DES FILAMENTS INTERMÉDIAIRES }\end{array}$} \\
\hline Type & Nom & Origine \\
\hline 1 & Kératines acides & Épithélium \\
\hline II & Kératines basiques & Épithélium \\
\hline III & Desmine & Muscle \\
\hline & Vimentine & Mesenchyme \\
\hline & GFAP & Astrocytes, cellules gliales \\
\hline & Périphérine & $\begin{array}{l}\text { Neurones du SNP, quelques neu- } \\
\text { rones du SNC }\end{array}$ \\
\hline & Plasticine & Rétine \\
\hline IV & Neurofilaments & Neurones \\
\hline & $\alpha$-internexine & Neurones du SNC \\
\hline V & Lamines nucléaires & Enveloppe nucléaire \\
\hline VI & Nestine & Cellules neuroépithéliales souches \\
\hline VII & Tanabine & Cône de croissance des neurones \\
\hline
\end{tabular}




\section{RÉFÉRENCES}

1. Weintraub $H$, Davis $R$, Tapscott $S$ et al. The myoD gene family : nodal point during specification of the muscle cell lineagc. Science $1990 ; 251: 761-6$

2. Pevny L, Simon MC, Robertson E et al. Erythroid differentiation in chimacric mice blocked by a targeted mutation in the genc for transcription factor GATA-1. Nature $1991 ; 349: 257-60$

3. Tronche F, Yaniv M. HNF1, a homoprotein member of the hepatic transcription regulatory network. BioEssays 1992; 14 : 579-87.

4. Steinert PM, Roop DR. Molecular and cellular biology of intermediate filaments. Ann Rev Biochem 1988 ; 57 : 593-625.

5. Lendahl U, Zimmerman LB, McKay RDG. CNS stem cells express a new class of intermediate filament protein. Cell 1990 ; $60: 585-95$.

6. Kaplan MP, Chin SSM, Fliegner $\mathrm{KH}$, Liem RKH. $\alpha$-internexin, a novel neuronal intermediate filament protein, precedes the low molccular weight neurofilament protein (NF-L) in the developping rat brain. I Neurosci $1990 ; 10: 2735-48$.

7. Hemmati-Brivanlou A, Mann RW, Har land RM. A protein expressed in the growth cones of embryonic vertebrate neurons defines a new class of intermediate filament protein. Neuron 1992 ; 9 : 417-28.

8. Glasgow E, Druger RK, Levine EM, Fuchs C, Schechter N. Plasticinc, a novel type III neurofilament protein from goldfish retina : increased expression during optic nerve regeneration. Neuron 1992 ; 9 : 373-81.

9. Greene LA. A new neuronal intermediate filament protein. TINS 1989; 12 : 228-30.

10. Portier M-M, Croizat B, Gros F. A sequence of changes in cytoskcletal components during neuroblastoma differentiation. FEBS lett $1982 ; 146$ : 283-8.

11. Thompson MA, Ziff EB. Structure of the gene encoding peripherin, an NGF regulated neuronal-specific type III intermediate filament protein. Neuron 1989 ; 2 : 1043-53.

12. Aletta JM, Shelanski ML, Greene LA Phosphorylation of the peripherin $58-\mathrm{kDa}$ neuronal intermediate filament protein. $J$ Biol Chem 1989 ; 264 : 4619-27.

13. Landon F, Lemonnier M, Benarous $\mathrm{R}$ et al. Multiple mRNAs encode peripherin, a neuronal intermediate filament protein. $E M B O J 1989 ; 8$ : 1719-26.

14. Troy CM, Muma NA, Greenc LA, Price DL, Shelanski ML. Regulation of peripherin and neurofilament expression in regenerating rat motor neurons. Brain Res

\section{Caractéristiques de la périphérine}

La périphérine, l'une des protéines du cytosquelette d'environ 57000 daltons, a été découverte en 1982 par M.M Portier et coll [10]. Toutefois, le gène de la périphérine n'a été isolé qu'en 1989 par Thompson et Ziff [11]. Il se compose de 9 exons séparés par 8 introns dont la structure coïncide exactement avec celles des gènes des FI de type III. Le gène humain de la périphérine est situé sur le chromosome $12[12 q 12-12 q 13]$ et le gène murin sur le chromosome 15 dans la région $[15 \mathrm{E}-15 \mathrm{~F}]$. La périphérine se trouve habituellement dans le corps cellulaire et l'axone du neurone. Comme pour les neurofilaments dont on rencontre les formes non phosphorylées dans le péricaryon et les dendrites - les formes phosphorylées étant plus abondantes dans les axones - le degré de phosphorylation pourrait intervenir pour contrôler la localisation de la périphérine dans le neurone. Trois isoformes phophorylées ont déjà été décrites et, en présence de NGF (nerve growth factor), la phosphorylation de la protéine augmente également [12]. La périphérine se renouvelle très lentement (demi-vie d'environ 7 jours) et son gène unique peut être transcrit en plusieurs ARN messagers d'environ 2 kilobases par épissage alternatif [13]. Les multiples isoformes de la périphérine proviennent donc à la fois de la traduction des différents ARN messagers et de modifications post-traductionnelles. Cependant, la signification précise de la diversité de ces isoformes reste à établir.

\section{Expression de la périphérine et différenciation}

Des chercheurs ont étudié en détail l'expression de la périphérine chez le rat $[16,17]$ et la souris [18]. Elle est présente dans la majorité des neurones dérivés de la crête neurale et dans certains neurones dérivés des placodes (neurones périphériques). On la trouve également dans les neurones moteurs de la corne antérieure de la moelle épinière, les noyaux moteurs des nerfs crâniens, ainsi que dans quelques autres noyaux du SNC (Tableau II) mais elle n'existe pas dans le cerveau antérieur. Enfin, son expression augmente durant la regénération des neurones $[14,15]$.

La périphérine est un excellent modèle pour étudier certains aspects
Tableau II

DISTRIBUTION DE LA PÉRIPHÉRINE

Tube neural

Encéphale *
Moelle épinière
Système optique

Noyaux moteurs des nerfs crâniens

Neurones moteurs de la corne antérieure Rétine et nerf optique

\section{SYSTĖME NERVEUX PÉRIPHÉRIQUE}

\section{Crête neurale}

Nerfs crâniens

Ganglions de la racine

dorsale

Nerfs rachidiens

Système nerveux

autonome

Fibres sympathiques

Fibres parasympathiques

Placodes

\section{Axones olfactifs \\ Ganglions acoustiques}

* Voir les références [19 et 20] pour des détails additionnels. 


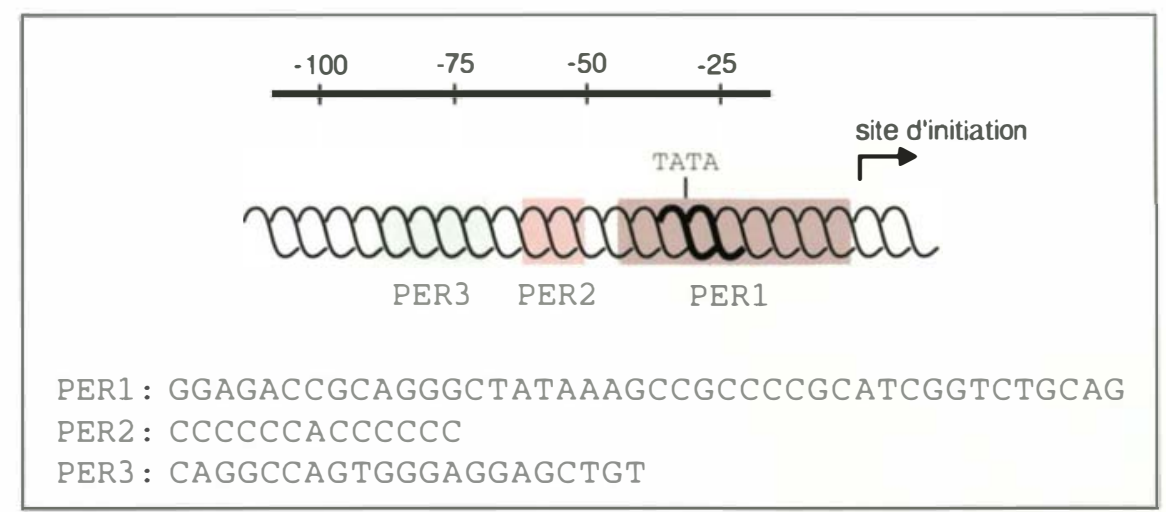

Figure 2. Promoteur du gène de la périphérine. Le promoteur contient 3 sites de liaison ADN-protéines, PER1, PER2 et PER3, dans la région qui s'étend jusqu'à la position -100. La séquence de ces éléments est indiquée sous le schéma.

du développement des SNP et SNC car son patron d'expression est limité à une sous-population de neurones. La production de neurones " matures " comprend trois phases: prolifération, migration et différenciation, pouvant être détectées chez l'embryon au moyen de la ségrégation spatiale des précurseurs. Dans la moelle épinière, la prolifération se produit dans la zone germinale adjacente à l'espace ventriculaire. Les précurseurs des neurones moteurs quittent le cycle mitotique et se déplacent au-delà de cette zone vers la partie ventrolatérale de la moelle épinière où aura lieu leur différenciation terminale, ainsi que l'axogenèse. Les neurones périphériques dérivés de la crête neurale ont une voie de maturation tri-

phasique similaire. Chez l'embryon de rat, au stade précoce de la neurulation (E10.5), la périphérine n'est pas décelable (hybridation in situ et immunohistochimie). Au stade E12.5, les cellules des lignages neuronaux ne sont pas toutes parvenues au même stade de maturation : quelques unes sont déjà post-mitotiques tandis que d'autres prolifèrent encore activement ou sont en phase migratoire. A ce stade, on a trouvé l'ARN messager du gène de la périphérine uniquement dans les neurones moteurs localisés dans la zone latérale de la moelle épinière et dans les neurones des ganglions de la racine dorsale. On ne le retrouve pas dans les régions à haute activité mitotique ou le long des routes de migration. Au cours du déve-

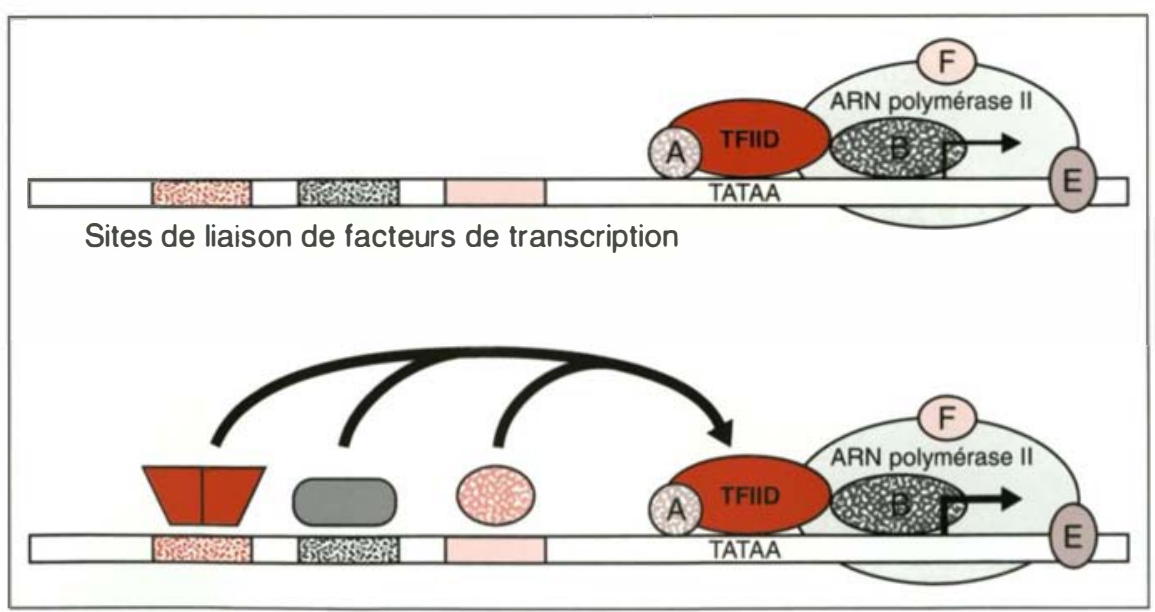

Figure 3. Organisation d'un promoteur typique. Les facteurs du complexe de pré-initiation (TFIID, TFIIA, TFIIB, TFIIE TFIIF) avec I'ARN polymérase II sont assemblés au niveau de la boîte TATA. L'activation transcriptionnelle est stimulée par des facteurs qui se lient plus en amont sur la séquence d'ADN et qui peuvent interagir avec un ou plusieurs facteurs du complexe de préinitiation.

$\mathrm{m} / \mathrm{s} n^{\circ} 5 \mathrm{vol} .9$, mai 93 loppement embryonnaire, la périphérine est détectée seulement lorsque les neurones périphériques et moteurs ont atteint leur position définitive. L'expression de la périphérine est donc étroitement associée à la différenciation neuronale. Toutefois, les connaissances actuelles ne permettent pas de dégager un thème fonctionnel ou développemental entre les divers neurons des SNP et SNC qui expriment la périphérine.

\section{Contrôle de la transcription du gène périphérine}

Le contrôle histospécifique de la transcription repose sur des interactions avec des facteurs qui se lient à proximité des gènes, très souvent dans la région qui les précède immédiatement. Le gène de la périphérine ne fait pas exception à la règle. Pour en faire la démonstration, un segment de 3500 pb adjacent à l'extrémité 5' du gène périphérine de souris, cloné dans notre laboratoire [21], a été fusionné à la séquence du gène de la $\beta$-galactosidase. La construction chimère a été transfectée dans des cellules en culture qui exprimaient ou non le gène de la périphérine. Ceci a permis de déterminer que le segment de $3500 \mathrm{pb}$ était suffisant pour le maintien de la spécificité neuronale d'expression. Une analyse plus précise effectuée au moyen de diverses constructions utilisant des séquences régulatrices de plus en plus courtes ou modifiées a permis la dissection fonctionnelle du promoteur et a démontré que les premières $98 \mathrm{pb}$ du promoteur suffisaient à assurer la spécificité neuronale d'expression in vitro [21]. La liaison de protéines à cette courte séquence d'ADN a été démontrée en utilisant des techniques d'empreinte à la DNAse I et d'essais de changement de mobilité électrophorétique. Ces moyens ont mis en évidence trois éléments appelés PER1, PER2 et PER3 (figure 2). La formation d'un complexe ADNprotéine avec l'élément PER 1 n'est observée qu'en présence d'extraits nucléaires provenant de cellules qui expriment la périphérine. Par contre, les éléments PER2 et PER3 semblent lier des protéines ubiquitaires. Ils ont cependant un rôle important à jouer 


\section{RÉFÉRENCES}

15. Wong J, Oblinger MM. Differential regulation of peripherin and neurofilament gene expression in regenerating rat $\mathrm{DRG}$ neurons. J Neurosci Res $1990 ; 27$ : 332-41.

16. Escurat $M$, Djabali $K$, Gumpel $M$, Gros F, Portier M-M. Differential expression of two neuronal intermediate-filament proteins, peripherin and the low-molecularmass neurofilament protein (NF-L), during the development of the rat. J Neurosci 1990 ; 10 : 764-84.

17. Gorham JD, Baker H, Kegler D, Ziff EB. The expression of the neuronal intermediate filament protein peripherin in the rat embryo. Dev Brain Res 1990 ; 57 : 235-48.

18. Troy CM, Brown K, Greene LA, Shelanski ML. Ontogeny of the neuronal intermediate filament protein, peripherin, in the mouse embryo. Neurosci $1990 ; 36$ : 217-37.

19. Leonard DGB, Gorham JD, Cole P, Greene LA, Ziff EB. A nerve growth factorregulated messenger RNA encodes a new intermediate filament protein. J Cell Biol $1988 ; 106: 181-93$.

20. Brody BA, Ley CA, Parysek LM. Selective distribution of the $57 \mathrm{kDa}$ neural intermediate filament protein in the rat CNS. J Neurosci 1989 ; 9 : 2391-401.

21. Desmarais D, Filion $M$, Lapointe $L$, Royal A. Cell-specific transcription of the peripherin gene in neuronal cell lines involves a cis-acting element surrounding the TATA box. EMBO J $1992 ; 11$ : 2971-80.

22. Greene LA, Tischler AS. Establishment of a noradrenergic clonal line of rat adrenal pheochromocytoma cells which respond to nerve growth factor. Proc Nall Acad Sci $1976 ; 73: 2424-8$.

23. Thompson MA, Lee E, Lawe D, Gizang-Ginsberg E, Ziff EB. Nerve growth factor-induced derepression of periperin gene expression is associated with alterations in proteins binding to a negative regulatory ele- dans la transcription du gène de la périphérine ; en effet, leur délétion diminue l'activité enzymatique de la $\beta$-galactosidase de $85 \%$. Ils n'ont toutefois pas d'activité "enhancer " intrinsèque. Soulignons enfin que les motifs nucléotidiques reconnus par ces protéines ne correspondent pas à des motifs de liaison de facteurs de transcription connus et que l'élément PER3 se retrouve dans tous les promoteurs des gènes des FI de classe III (périphérine, vimentine, desmine et GFAP). Cette constatation invite à envisager l'existence d'une nouvelle famille de facteurs de transcription. Le promoteur est donc composé de plusieurs modules. Parmi ces éléments, PER1 occupe toutefois une place à part. Cette séquence, qui comprend la boîte TATA, n'a aucune activité par elle-même mais elle est suffisante pour conférer la spécificité neuronale d'expression lorsque le "enhancer" du virus du Polyome, activateur qui fonctionne dans toutes les cellules, lui est accolé. En comparaison, PER2 et PER3 ont une activité négligeable lorsqu'ils sont recombinés à un autre promoteur et, surtout, ne montrent aucune histospécificité. PER1 apparaît donc comme l'élément central du promoteur du gène périphérine.

La position stratégique de PER 1 soulève notamment la question de sa relation avec le complexe de pré-initiation et tout particulièrement avec TFIID, le complexe protéique qui se lie à la boîte TATA. Les modèles habituels évoqués pour expliquer l'interaction des facteurs de transcription (figure 3) pourraient ne pas s'appliquer ici. En effet, le complexe protéique liant l'élément PER 1 n'est un activateur pas plus dans le promoteur du gène de la périphérine que dans un autre contexte. De plus, la présence de l'élément PER 1 est suffisante pour restreindre l'activité d'un "enhancer " aussi fort que celui du virus du Polyome aux cellules qui expriment la périphérine. Il est donc probable que PER 1 ait une structure qui empêche l'interaction de TFIID avec la boîte TATA ou encore que PER 1 favorise l'établissement ou le maintien de la chromatine dans une conformation transcriptionnellement inactive. Comment fonctionne PER1 ? Il pourrait agir comme un "verrou de sécurité " empêchant certains éléments ou facteurs d'activer le gène de façon inopportune ; la clé de ce "verrou " serait le facteur qui lie l'élément PER1. Deux modèles semblent envisageables (figure 4). Dans le premier modèle, le complexe protéique liant l'élément PER1, le complexe X (figure 4a), se lie de façon transitoire à PER 1 et permet ou stabilise la liaison de TFIID à la boîte TATA. Dans le deuxième modèle (figure 4b), le complexe $\mathrm{X}$ modifie TFIID pour permettre la formation d'un complexe transcriptionnel actif avec les protéines qui lient les éléments PER2 et PER3. Dans une variante de ce modèle, le complexe $\mathrm{X}$ modifierait une des composantes du complexe de pré-initiation. Les résultats obtenus jusqu'à maintenant ne permettent pas de distinguer lequel est le plus fidèle à la réalité. Ils soulignent toutefois clairement le rôle central de PER 1.

Le déroulement d'un programme de différenciation cellulaire ne repose cependant pas uniquement sur des facteurs de transcription histospécifiques. Il fait aussi entrer en jeu des facteurs environnementaux. Le système cellulaire le plus utilisé pour étudier la différenciation neuronale est la lignée PC12. Cette lignée se différencie en neurones sympathiques en présence de NGF [22]. Cet état se traduit par une augmentation de la transcription de plusieurs gènes, dont celui de la périphérine. Une étude récente [23] laisse supposer que le mécanisme de la réponse au NGF du gène de la périphérine serait biphasique et exigerait le retrait d'un répresseur situé entre les positions -178 et -168 afin de permettre à un activateur situé plus en amont de stimuler la transcription.

Ces travaux illustrent donc comment plusieurs facteurs de transcription différents peuvent, en agissant de concert, limiter l'expression du gène de la périphérine à un type cellulaire défini et moduler le degré de son expression. Comme ces facteurs de transcription contrôlent certainement plusieurs gènes, l'isolement et la caractérisation des facteurs qui contrôlent l'expression de la périphérine ainsi que son induction par le NGF promettent de révéler des facteurs importants pour le développement et la différenciation des neurones 


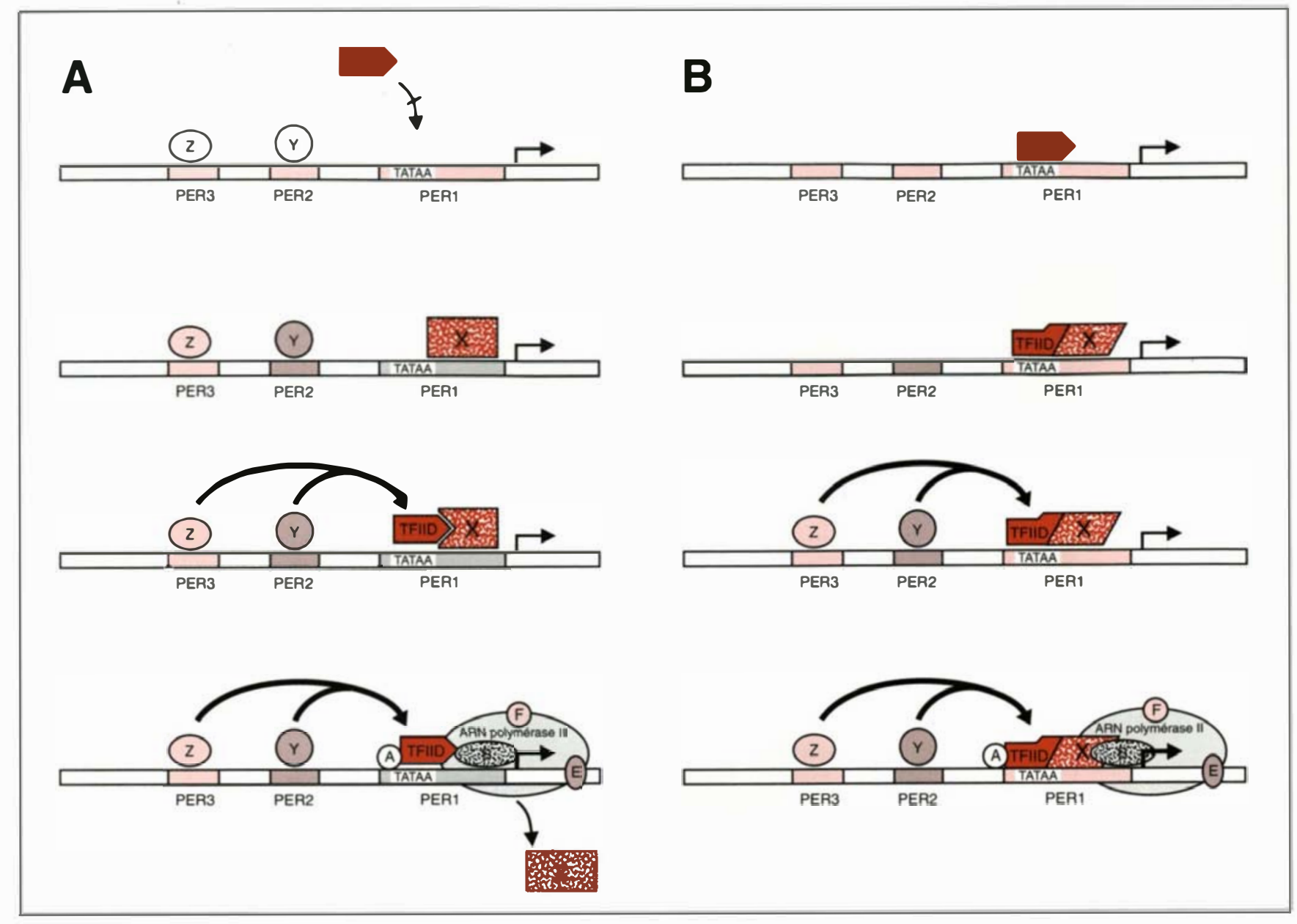

Figure 4. Représentation schématique de l'activation du gène de la périphérine. A) la liaison de la protéine $X$ à l'élément PER1 permettrait de stabiliser la liaison du complexe TFIID à I'ADN. Cet état permettrait ensuite à d'autres facteurs et au complexe de pré-initiation de lier l'ADN et $d^{\prime}$ activer le gene. B) La protéine $X$ une fois liée à PER1 serait en mesure de modifier TFIID par un mécanisme qui reste à déterminer, qui pourrait certainement mettre en jeu soit une liaison protéine-protéine soit une modification enzymatique.

TIRÉS A PART

A. Royal.

$m / s n^{\circ} 5$ ool. 9, mai 93

\section{Summary}

Intermediate filaments and neuronal gene expression

In the developing nervous system, intermediate filaments have a specific and diversified pattern of expression. In particular, peripherin is expressed only in neurons and mostly in the peripheral nervous system where its expression is associated with terminal differentiation. Molecular analysis of the promoter of the peripherin gene revealed that the 98 base pairs preceding the transcription start site were sufficient to confer cell-type-specific expression. Three regulatory sequence elements named PER1, PER2 and PER3 were found in this region. Experiments designed to test the contribution of each element to the regulation of peripherin gene transcription showed that PER2 and PER3 were important in determining the level of expression, while PER 1 was important for cell-type specificity. The PER 1 element is also particular in that it overlaps the TATA box and interacts with a DNA-binding protein found in peripherin expressing cell lines. These observations indicate that cooperative interactions between different regions of the promoter are necessary for efficient and cell-type-specific transcription of the peripherin gene in a subset of neuronal cells. PER 1 also identifies a novel neuron-specific transcription factor. 